INSTITUTO DE INVESTIGACIONES CIENTÍFICAS (IDIC)

SECRETARÍA DE POLÍTICAS DEL CONOCIMIENTO

FACULTAD DE ARTE, DISEÑO Y COMUNICACIÓN

UNIVERSIDAD DE LA CUENCA DEL PLATA.

CORRIENTES, ARGENTINA

BURGOSCARLOS_CEN@UCP.EDU.AR

INSTITUTO DE INVESTIGACIONES CIENTÍFICAS (IDIC)

SECRETARÍA DE POLÍTICAS DEL CONOCIMIENTO

FACULTAD DE ARTE, DISEÑO Y COMUNICACIÓN

UNIVERSIDAD DE LA CUENCA DEL PLATA.

ROMINA-FAURE-852@HOTMAIL.COM.
CARLOS EDUARDO BURGOS

ROMINA FAURE

CORRIENTES, ARGENTINA

\section{Estructuras reticulares en los modelos metodológicos de las disciplinas proyectuales}

Reticular structures in the methodological models of design disciplines

Resumen. Este trabajo aborda una problemática abierta en la agenda de investigación de los estudios de diseño (design studies): la búsqueda de modelos metodológicos comunes a diferentes tipos de actividad proyectual, alejados de los modelos metodológicos clásicos que conciben las distintas expresiones del diseño como compartimentos estancos. Se analizan procesos cognitivo-operativos implicados en el diseño, traduciéndolos en redes de decisiones, actores y actantes (concebidos como agencias), tomando como casos de estudio el diseño gráfico y el diseño de indumentaria-textil. Este objeto de estudio se define, en particular, por sus sistemas de relaciones, los objetos que producen, las estrategias que utilizan y las ideas dominantes de cada actividad. La estrategia general de la investigación combina la construcción de un aparato crítico conceptual con un diseño empírico descriptivo utilizando una técnica de observación de prácticas de campo conocida como Análisis de Protocolo de Réplica. Los resultados muestran algunos rasgos preliminares de un modelo metodológico común a diferentes expresiones proyectuales, y el carácter complejo de sus procesos -reticulares- de constitución. Se aporta una conceptualización metodológica centrada en la idea de 'agencia' $y$ 'red proyectual' como un entramado de actores, actantes y discursos presentes en los procesos implicados en las diferentes expresiones proyectuales analizadas.

Palabras clave: agencias, diseño, metodología, redes proyectuales.

Este trabajo forma parte del proyecto acreditado por la Universidad de la Cuenca del Plata -UCPArgentina, denominado "Las redes estratégicas como mecanismos de innovación en los modelos metodológicos de las disciplinas proyectuales", aprobado y financiado por Resolución 281/16 y radicado en el Instituto de Investigaciones Científicas (IDIC), Secretaría de Políticas Universitarias de la UCP-Argentina.

Fecha de recepción: 10/11/2017

Fecha de aceptación: 23/05/2018

Cómo citar: BURGOS, C. Y FAURE, R. (2018)

Estructuras reticulares en los modelos metodológicos de las disciplinas proyectuales. RChD: creación y pensamiento, 3(4), 1-12. DOI: $10.5354 / 0719-837 X .2018 .50237$

Revista Chilena de Diseño

RChD: creación y pensamiento

Universidad de Chile

2018, 3(4)

http://rchd.uchile.cl
Abstract. This study addresses a current issue in the design studies research agenda: the search of cross-methodological models that can explain different kinds of design activities, which are distant from the rigid conventional disciplinary boundaries. Cognitive and operative processes are analyzed and translated into a network of actors and actants in the different modalities of the design praxis, using as study cases graphic, and garments-textile design. Their relationship systems, the objects they produce, the strategies they use and the dominant ideas of each activity define this issue. The research strategy combines the construction of a critical conceptual framework with a descriptive empirical design utilizing a fieldwork observation technique called Replication Protocol Analysis. The results show preliminary aspects of a common methodological model to different projective expressions and the complex nature of their constitutional process.

A methodological conceptualization is provided; centered on the idea of "agency" and "projecting network" as a set of actors, actants and discourses present in the processes of the different design expressions that were analyzed.

Keywords: agencies, design, methodology, projecting networks. 
Figura 1. Pasos o etapas en el modelo metodológico clásico. Fuente: Visser 2006, p. 34.

1. Consideramos que estos autores continúan el legado de la generación de investigadores de los "nuevos métodos", de la década de los sesenta que definieron las bases de una concepción todavía dominante (Alexander, 1964; Gregory, 1966; Archer, 1984; Broadbent, 1984; Simon, 1988; Jones 1992)

\begin{tabular}{|c|c|c|c|c|c|c|}
\hline Author or Source & \multicolumn{2}{|c|}{ Stage I } & Stage 2 & \multicolumn{3}{|c|}{ Stage 3} \\
\hline Simon (1996) & $\begin{array}{r}\text { Stru } \\
\text { (if ill-struct }\end{array}$ & $\begin{array}{l}\text { uring } \\
\text { d problem) }\end{array}$ & Problem solving & & & \\
\hline Hamel (1989) & \multicolumn{2}{|c|}{ Analysis } & Synthesis & \multicolumn{3}{|c|}{ Molding } \\
\hline $\begin{array}{l}\text { Classical Cognitive } \\
\text { Psychology }\end{array}$ & \multicolumn{2}{|c|}{$\begin{array}{c}\text { Problem - representation } \\
\text { construction }\end{array}$} & Solution generation & \multicolumn{3}{|c|}{ Solution evaluation } \\
\hline Schön & Naming & Framing & Moving & \multicolumn{3}{|c|}{ Evaluation } \\
\hline De Groot & \multicolumn{2}{|c|}{ Perception / observation } & Trying & \multicolumn{3}{|c|}{ Evaluation } \\
\hline Jones & \multicolumn{2}{|c|}{ Analysis } & Synthesis & \multicolumn{3}{|c|}{ Evaluation } \\
\hline VDI Guideline 222 I & $\begin{array}{l}\text { Problem } \\
\text { analysis }\end{array}$ & $\begin{array}{l}\text { Problem } \\
\text { definition }\end{array}$ & System synthesis & $\begin{array}{l}\text { System } \\
\text { analysis }\end{array}$ & $\begin{array}{l}\text { Evalua- } \\
\text { tion }\end{array}$ & $\begin{array}{l}\text { Decision } \\
\text { making }\end{array}$ \\
\hline
\end{tabular}

\section{Introducción}

La imagen dominante sobre la metodología del diseño presenta un escenario teórico de múltiples actividades tabicadas según disciplinas que definen al diseño en el marco de un expertice (Lawson, Dorst 2009; Cross 2010; Cross 2011). Esta fragmentación no permite avanzar en una comprensión más profunda de sus fundamentos, metodologías e impactos culturales, a partir de una visión más integradora y transversal respecto de diferentes tipos de práctica (Burgos 2010, 2013). Indagamos la condición transdisciplinar del diseño, constituida por un conjunto de actividades que comparten el mismo espacio de problemas (proyectuales) y similares sistemas de producción metodológica. Desde este escenario analizamos -utilizando la teoría del Actor-Red- los modelos metodológicos comunes implicados en diferentes procesos de diseño, buscando, a partir de la especificidad de cada expresión proyectual, generar un esquema común con base en la pluralidad y diversidad de agencias que participan del fenómeno proyectual ampliado.

Este enfoque intenta producir un avance en el abordaje de los procesos de diseño en diferentes disciplinas proyectuales, tales como el diseño gráfico y el de indumentaria-textil. Su punto de partida tiene anclaje en la cuestión instalada por Visser sobre el fundamento común que poseen todos los diseños: "design is one, but in different forms" (2009, p. 187). Esta idea ha dado base a pensar en la existencia de cuestiones comunes entre las distintas expresiones de lo proyectual, tanto en el plano onto-epistémico como en el metodológico. Sin embargo, en los debates sobre la cuestión del método de diseño aún puede advertirse cierta preeminencia de planteos centrados en "pasos", "etapas" o "fases" para la toma de decisiones. Es posible, también, observar un sesgo hacia una dimensión prescriptiva del proceso, con referencia a estructuras secuencialistas, jerárquicas y lineales, como las presentes en las 'recetas' clásicas de autores tales como Munari (2006), Frascara $(2010,2018)^{1}$ o Cross (2008). Un análisis de los principales componentes presentes en estos modelos expone la prevalencia de estructuras metodológicas centradas en instancias decisionales sucesivas, que distinguen momentos de análisis y momentos de creación; procesos lógicos e instancias creativas; etapas informativas y propositivas, etc.; Dubberly (2004); Curry (2014).

En el esquema que sigue (Ver Figura 1), Visser (2006) sintetiza el contenido de los modelos principales que perviven en la concepción actual. 
Este escenario valida y refuerza la fragmentación del territorio proyectual y parcela el proceso en etapas, habilitando una división o distinción fuerte entre diferentes campos disciplinares. En este sentido, pareciera ser posible afirmar que el diseño gráfico podría tener poco que ver con el diseño de indumentaria o con el diseño arquitectónico. Los productos de estas prácticas son distintos, aunque poseen un rasgo común: son artefactos socio-técnicos que, aunque diferentes en forma y función, operan de modo similar en la interface vinculada con los procesos de adaptación y transformación del mundo a través de la producción de objetos-artefactos tecnológicos.

En este sentido, consideramos vigente la concepción ampliada o expandida sobre la producción de objetos de autores como Hutchins (1996) o Hacking (1996), ${ }^{2}$ con los cuales podemos considerar la práctica del proyecto como una interacción de agencias (actores, actantes y discursos) y no como un conjunto de acciones independientes o aisladas unas de otras. Se entiende, así, a las prácticas de diseño en un contexto cultural expandido, más que en uno disciplinar-profesional restringido. Mantener una posición disociada entre las diferentes manifestaciones del diseño impide explotar los posibles 'diálogos' que se producen entre ellas y avanzar en la comprensión profunda de sus argumentos fundamentales (Burgos y lbarra, 2015).

Aún si consideramos la definición clásica de Simon, "Everyone designs who devises courses of action aimed at changing situations into preferred ones [...]. Design, so construed, is the core of all professional training" (1996, p. 55), podemos analizar el modo en que se constituyen estos cursos de acción y que tipo de procesos o mecanismos los alimentan. Desde este enfoque, también es posible preguntarse ¿cuál es la relación que existe entre diferentes prácticas proyectuales (gráficas, de indumentaria, textiles, industriales, arquitectónicas, etc.) derivadas de sus procesos de concepción, diseño y producción? ¿Qué estructuras transversales o comunes es posible explorar en sus correspondientes objetos, metodologías y resultados, que son condición de posibilidad de la innovación socio-técnica que plantean? ¿Qué rol juegan los procesos cognitivos de la práctica? Y muchas otras que exceden el contenido de esta contribución.

El supuesto base que alimenta esta investigación es la existencia de fundamentos y mecanismos comunes en los procesos metodológicos de las disciplinas proyectuales, dado que comparten una misma base creativa tanto cognitiva como tecnológica- y un mismo escenario de producción socio-técnico. ${ }^{3} \mathrm{~A}$ partir de esta idea y del análisis de diferentes tipos de praxis proyectual, se puede indagar en los modelos metodológicos centrados en la condición representacional ${ }^{4}$ de su lenguaje y en la pertenencia a estructuras reticulares de agencias, actores y actantes (Latour, 1997; Callon, 1987). De este modo, proponemos pasar de una idea de método como programa preestablecido a la de un proceso estratégico dinámico (Morin, Ciurana et al., 2003), que se configura en movimiento sobre la base de agencias o dimensiones tanto humanas como técnicas.

\section{Metodología}

Este trabajo se ha focalizado en la praxis del proceso de diseño en un registro epistémico que intenta detectar la generación de conocimiento en-el-proceso-mismo-de-la-práctica proyectual. Dentro de este marco, se ha propuesto avanzar en una comprensión metodológica/praxeológica del saber implicado en el proceso, consistente con las prácticas efectivas que
2. Hutchins y Hacking provienen de la filosofía de la ciencia y la tecnología, y sus aportes son válidos para comprender los procesos del diseño. En particular, el concepto de conocimiento "expandido" de Hutchins permite comprender los objetos culturales como portadores de estructuras cognitivas y lógicas de significación. Hacking plantea que conocer e intervenir son dos momentos de un mismo proceso productivo, cuestión que permite pensar en el diseño en un plano tecnológico, pero también epistémico. Estas ideas dan pie para pensar en un "territorio" común a diferentes prácticas proyectuales, tal como se expone en esta contribución.

3. El uso de la concepción socio-técnica tuvo una amplia difusión luego de la publicación del trabajo de Bijker, Hughes y Pinch en 1987 (The Social Construction of Technological Systems: New Directions in the Sociology and History of Technology), en la que ambos campos -social y tecnológico- se entretejen en diferentes expresiones humanas. En este artículo, esta base es importante para sostener el enfoque general de la presentación.

4. El concepto de representación usado aquí indica la capacidad de los diseñadores de traducir agencias (ideas, necesidades, usuarios, pautas o discursos) en formatos gráficos que logran preservar la lógica de los contenidos que representan, aunque en formatos diversos. Por ejemplo, un organigrama traduce a gráfico toda una red de conexiones entre diferentes espacios, condicionados por la función, las necesidades, los usos y las actividades. 
Figura 2. Reconstrucción gráfica del modelo clásico desarrollado a partir de la segunda mitad del siglo XX y que aún pervive en nuestras concepciones metodológicas. Fuente: Jones (1992).

5. Estas estrategias, si bien producen resultados diferentes, se integran en una sola conclusión, ya que permiten observar el 'objeto de estudio' desde diferentes perspectivas, aunque intentando aportar una visión integrada del fenómeno analizado.

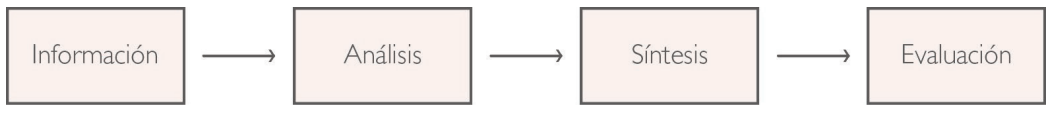

los diseñadores llevan adelante en su labor cotidiana y desarrollar, con ello, categorías teórico-metodológicas apropiadas para elucidar estructuras y/o dinámicas comunes. Para ello se ha diseñado una triangulación de tres estrategias metodológicas concurrentes ${ }^{5}$ (una filosófico-crítica y dos empírico-descriptivas):

i) Un relevamiento del estado de la cuestión sobre las categorías más relevantes que la investigación disciplinar y las publicaciones vigentes proponen en el campo de la metodología de las disciplinas proyectuales.

ii) Aplicación de una estrategia cuasi-experimental sobre los productos del proceso de diseño (protocolos) y sus correspondientes instancias de razonamiento lógico, mediante la técnica de Análisis de Protocolo de Réplica-RPA-Protocol (Galle, 1996), a partir de los dibujos, diagramas, notas, argumentos, audios, etc., que capturan diferentes procesos simbólicos del proyecto.

iii) Un análisis comparativo de los principales modelos metodológicos del diseño gráfico, de indumentaria y textil, utilizando los componentes del paradigma del actor-red ya mencionado, que permite asimilar los procesos detectados a una trama de actores, actantes y discursos.

\section{Discusión de resultados}

El estudio generó dos tipos de resultados: a) Un planteamiento conceptual apropiado para orientar la construcción de una imagen diferente del fenómeno proyectual y, b) Algunos elementos comunes detectados en los casos de estudio (diseño gráfico, indumentaria y textil) con los que se configuró un esquema o concepción preliminar alternativa para la metodología del diseño en un registro reticular-complejo y no jerárquico-secuencial.

\section{Revisión del marco conceptual de la metodología del diseño}

Los planteos metodológicos aplicados al análisis de las disciplinas proyectuales conservan todavía un sesgo determinista en sus enfoques. Aún persisten los modelos jerárquico-lineales en los que se representan tareas o etapas poco consistentes con las prácticas reales que llevan adelante los diseñadores. Los "mapas" o itinerarios que propone la metodología convencional son secuencialistas y jerárquicos, sugiriendo tabiques entre tareas creativas, evaluativas, informativas y productivas (Cross 2008, Frascara 1988, Munari 1989, Jones 1992). Estos aportes representan el núcleo de una concepción ya clásica sobre la metodología que se construye (con variantes) a partir de cuatro etapas: (ver figura 2). Con el fin de contrastar este esquema proponemos pensar en el territorio del diseño no como un proceso lineal sino como una red. En este registro, las prácticas que realiza el diseñador se concentrarían en relacionar y articular cuestiones, actores y aspectos diversos. La investigación mostró que los diseñadores van focalizando su práctica en algún aspecto, elemento o situación y esta lo lleva a otra, con la que establece una interacción estratégica o de interdependencia. Ha sido evidente que tal proceso no se puede representar con una línea, sino con una malla o red de nodos (aspectos) y relaciones (vinculaciones) en una dinámica de construcción compleja. 
Un modelo gráfico de esta idea se observa en la Figura $3 .{ }^{6}$

Con similar inspiración, otros estudios de investigación en el campo (Buchanan, 1992; Roth, 1999; Margolin, 1989; Morelli, 2007; Visser, 2006, 2009; Farrell y Hooker, 2013) revelan la inadecuación de los enfoques clásicos y orientan a un nuevo camino de abordaje para elucidar las prácticas proyectuales y los alcances que se derivan de sus procesos constitutivos, promoviendo la posibilidad de avanzar hacia modelos transdisciplinares que intenten comprender su impacto en la configuración de la cultura (también entendida como la concepción y planificación de lo-artificial). Asimismo, otras fuentes introducen el concepto de agencia e intentan repensar las bases teóricas del diseño, aunque reconociendo la base tradicional que pervive en la actualidad (Kimbell, 2011, 2012; Kokotovich y Dorst, 2016; Pierri, 2017).

Por otra parte, la concepción sobre la influencia de la cultura de lo artificial (Simon 1988, Margolin, Morales et al. 2007) posee un amplio consenso dentro de una comunidad de investigación que ha trabajado por muchos años sobre este concepto como un eje fundamental para definir objetos de estudio y metodologías de abordaje de la cuestión proyectual. Desde este punto de vista sobre el rol del diseño en el contexto social, Buchanan afirma que "they are drawn together because they share a mutual interest in a common theme: the conception and planning of the artificial" (Buchanan, 1992, p. 14). En este contexto, planificar el mundo 'artificial' es tema de trabajo de muchas disciplinas diferentes en el ámbito del diseño. Nos proponemos indagar en este espacio 'expandido' de proyecto desde el análisis de las prácticas del diseño gráfico, el diseño de indumentaria y textil.

Algunas de estas cuestiones están ya en germen en la posición más influyente en el territorio del diseño desarrolladas por Simon (1996, p. 55). Sin embargo, esta concepción ha llevado a la construcción de una visión teórico-metodológica dominante para el diseño que no logra dar cuenta de la verdadera complejidad de la actividad proyectual y del rol que juega en el complejo escenario de la cultura actual. El eje profesionalista de su planteo y su consecuente orientación hacia la resolución de problemas "típicos" del mundo no ha permitido la apertura de la actividad de diseño hacia nuevas dimensiones de su complejidad, y del modo en que el conocimiento (científico), la tecnología y la producción la afectan especialmente.

En una cartografía clásica de problemas de investigación sobre el escenario del diseño, Cross $(1982,2007)$ analiza una taxonomía posible para los problemas (de investigación) de diseño, con una afirmación que define, en gran medida, todo el programa dominante de la investigación actual, al afirmar que el conocimiento implicado en el diseño reside primero en las personas (diseñadores) luego en los procesos (métodos) y, finalmente, en los productos (Cross 1999). De ahí que, para esta visión dominante, todo el fenómeno proyectual está determinado por cierta habilidad especial que los diseñadores poseen (expertiz) y que define la naturaleza epistémica y metodológica de la actividad. Los conocimientos proyectuales se radican también en los procesos, es decir, "in the tactics and strategies of designing. A major area of design research is methodology: the study of processes of design, and the development and application of techniques which aid the designer" (Cross, 1999, p. 6). El tercer integrante del escenario problemático es el producto, asumido solo desde su condición material: "in the forms and materials and finishes which embody design atributes" (Cross, 1999, p. 6), y no como vehículo de estructuras cognitivas. Estas precisiones sobre el campo de investigación lo han

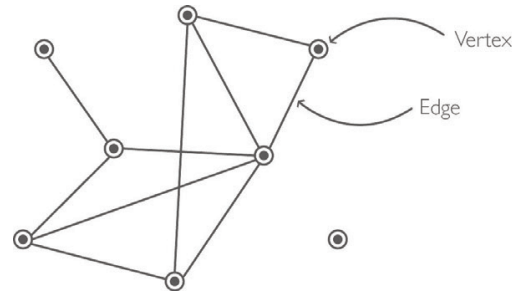

Figura 3. Nodos y conexiones de una red.

Fuente: Newman, 2003, p. 169.

6. En este esquema se observa una estructura típica de nodos o vértices y conexiones o bordes. Estos componentes pueden variar en cada proceso de diseño, aunque la estructura de relaciones e interconexiones es lo que pretendemos exponer como una estructura invariante. 
llevado a definir una taxonomía de problemas basada en personas, procesos y productos, aplicables a tres categorías básicas: i) Design epistemology (study of designerly ways of knowing); ii) Design praxiology (study of the practiced and processes of design); iii) Design phenomenology (study of the form and configuration of artefacts) (Cross 2007, pp. 125s.).

En el caso de la epistemología del diseño, Cross propone el avance sobre la construcción de una visión que definiría cuáles son sus procesos. Finalmente, la fenomenología se abocaría a los rasgos y atributos de la configuración material de los artefactos, en tanto entidades físicas dotadas de una funcionalidad técnica y una estética formal.

Desde otro punto de vista, aunque dentro del mismo paradigma conceptual, Margolin plantea un escenario de temas de investigación relacionados con el diseño como actividad dinámica, con instancias de modificación y cambio en sus métodos, discursos y productos. Así, propone un espacio de problemas vinculados con 4 enfoques principales (Margolin 2007, pp. 31s.):

i) El estudio de las prácticas del diseño, perteneciente al dominio de la acción de los sujetos que llevan adelante la práctica (diseñadores). Son exponentes de estos problemas los trabajos de autores como Schön (1992) o Cross, Christiaans y Dorst (1996).

ii) El estudio de los productos de diseño, centrado en las características formales y funcionales de los objetos. Las perspectivas de análisis son variadas ya que los objetos pueden ser vistos desde múltiples perspectivas. En esta línea podemos mencionar los trabajos de autores que han incorporado la filosofía de la tecnología al análisis de los productos del diseño (Houkes, 2006; Kroes y Meijers, 2002; Lawson y Dorst, 2009).

iii) El estudio de los discursos (teórico-filosóficos) sobre el diseño, que apuntan a definir lo que es el diseño, en la encrucijada entre la técnica, la ciencia y el arte. Son enfoques principalmente epistemológicos, como en el caso de Galle (1999, 2002), Margolin (1989) o Buchanan (1985).

iv) Por último, el estudio de las metodologías, en el que los textos abordan, con profusión, diferentes rasgos de los metadiscursos (reconstrucciones racionales) que se construyen sobre la actividad proyectual con perfiles básicamente prescriptivos, deterministas y secuenciales (Jones, 1992; Archer, 1981; Kroes, 2002; Jonas, 1993; Jacques y Powell, 1980; Cross 1984). Finalmente, algunos autores han destacado la necesidad de una convergencia de los estudios sobre el diseño superando la existencia de tabiques en su territorio (Buchanan y Margolin, 1993, Margolin, Morales et al., 2007).

Por todo ello, consideramos importante orientar la búsqueda hacia un diálogo constructivo y estratégico con todas las actividades que comparten los mismos problemas y fundamentos proyectuales -en tanto actividad socio-técnica-, a fin de generar un abordaje más potente para dar cuenta de los procesos de transformación de las entidades tecnológicas, cognitivas y sociales que dan forma a la vida actual.

Sobre estos aportes y antecedentes se centra el objeto de esta investigación y su marco conceptual, a fin de configurar nuevos modelos 'metodológicos' ubicados en la interface o 'interferencia' de las diferentes praxis constructivas de las disciplinas proyectuales en un registro (trans) disciplinar e intersectorial. 


\section{Aspectos comunes a diferentes expresiones proyectuales}

A partir de un primer análisis del proceso de diseño centrado en cada disciplina, se detectaron actores comunes, mecanismos de trabajo y sistemas de relaciones similares. La estrategia de traducir los elementos diversos a la condición de agencias llevó a pensar que el entramado de las conexiones entre actores y actantes era lo que determinaba y sustentaba la formulación, análisis y posible resolución de los problemas de diseño. Esta primera conclusión permitió elaborar instancias de síntesis de los diferentes esquemas analizados, a partir de cuatro núcleos organizadores de pares de actores-actantes, sin que ello sea una combinación rígida o cerrada ya que los mismos fueron reconfigurándose de acuerdo al momento del proceso en que se encontraron, advirtiendo la existencia de un mecanismo de diseño con instancias de trabajo iterativas o de "ida y vuelta".

En definitiva, estos núcleos se definieron como: Usuario-Cliente; Escenarios-Productos; Ciencia/Tecnología-Materialidad; Diseñador-Estrategias. ${ }^{7}$

\section{a) Usuario-Cliente}

Se observó que las demandas de los clientes actuaron como un disparador de acciones metodológicas, sea esta detectada por el mismo cliente o visualizada en el análisis de las potenciales demandas del usuario; entendiendo al usuario como aquella persona que usa algo para una función específica en la satisfacción de alguna necesidad, y al cliente como aquel que solicita o encarga el servicio-producto. Ambos poseen requerimientos e intereses que determinaron su participación activa en el proceso, y que se convirtieron en fundamentos para la toma de decisiones, permitiendo canalizar -a través de ellos- un conjunto de relaciones e interacciones entre diferentes aspectos. Esta cuestión ha sido vital, a fin de que el producto-servicio resultante del proceso satisfaga las necesidades y demandas de ambos. Si la concepción del problema no parte o se concibe a partir de ellos, poco podrán aportar las siguientes etapas de desarrollo a la aceptación del producto por parte del usuario final. Tanto cliente como usuario se presentaron condicionados por un contexto complejo, histórico o coyuntural, a partir del cual fue posible asignar sentido a las necesidades, los valores, los intereses y los objetivos de la acción de diseño. Los diseñadores orientaron sus metodologías de trabajo hacia la satisfacción de los requerimientos del cliente y de las necesidades del usuario, pretendiendo conseguir la mayor satisfacción y mejor experiencia de uso posible con el mínimo esfuerzo.

\section{b) Escenario-Productos}

Cuando hablamos de escenarios nos referimos a la visión que expusieron los diseñadores de múltiples espacios o situaciones en la que sería posible el consumo, uso o impacto del producto de diseño, concibiéndolo como un "encuentro" de condiciones de adaptabilidad y cualidades de los objetos de diseño, que posibilitan que el 'artefacto' pueda 'insertarse' en el ámbito cultural (social, político, económico, etc.) para el cual ha sido creado. Crear escenarios fue una estrategia que permitió a los diseñadores pensar el producto en un contexto determinado, trazando estrategias de resolución concretas. El contexto sociocultural no es susceptible de ser modificado y fue altamente influyente en la percepción de la situación de intervención que definieron los diseñadores. Conocer el escenario en el cual se insertará el producto permitió definir y ajustar con precisión los requerimientos del usuario.
7. Estos aspectos fueron relevados de los procesos desarrollados por los diseñadores. En sus diferentes prácticas fueron relevantes tanto en los discursos como en los productos (protocolos). 
También se ha observado que las propuestas finales estuvieron condicionadas por experiencias anteriores de los diseñadores, instancias en las que ya ha podido establecer relaciones de influencia o fundamentación. Cuanto mayor ha sido el grado de experticia del diseñador mejor ha sido su capacidad para configurar el análisis de la problemática concreta en la que le tocó actuar y desarrollar miradas críticas en la generación de estrategias innovadoras: nuevas tecnologías o modos de hacer, avances científico-técnicos, escenarios de trabajo cambiantes, obsolescencia de ciertas metodologías, etc.

c) Ciencia/Tecnología-Materialidad La aproximación a la ciencia y tecnología se ha observado al momento de advertir cuáles fueron los materiales y las técnicas necesarias para el trabajo del diseñador, desde la percepción del escenario actual para la comprensión del problema y hasta las posibilidades brindadas por nuevos avances en las formas de representar o modelizar. Las herramientas de digitalización, representación y modelización adquieren una relevancia clave en la estrategia comunicacional. Por otra parte, el conocimiento de nuevos insumos y procesos de producción industrial ha condicionado fuertemente la puesta a prueba de las ideas generadas y los resultados esperados.

Asimismo, ha sido clave la importancia del equipamiento como herramienta de trabajo del diseñador, determinando en parte los procesos que se estudiaron durante las diferentes instancias proyectuales, desde la investigación hasta la puesta en marcha de la estrategia final.

El equipamiento como parte de la estrategia de resolución del problema determinó, además, las limitantes para una propuesta factible de ser realizada, tanto desde los costos y los tiempos, hasta las posibles relaciones con los proveedores y la disponibilidad de los materiales e insumos seleccionados.

d) Diseñador-Procesos

El proceso de diseño permite una alta implicación del diseñador, de sus ideas, lenguajes, valores y formas de trabajo. La instancia de generación de pautas, objetivos e intenciones dejó en claro el modo en que la experiencia-experticia de los diseñadores permitía conducir con mayor solvencia el proceso. En este sentido, el diseñador es el "actor" clave que establece las interconexiones entre las demás agencias, haciendo valer sus marcos teóricos y lógicos en la definición de los discursos que acompañan las propuestas y les otorgan fundamento y sentido.

En cuanto al proceso, no se advirtió que este siguiera la fórmula canónica Información-análisis-síntesis-evaluación, por el contrario, las decisiones parecían registrar diferentes aspectos al mismo tiempo y en diferentes niveles de complejidad. El proceso no se manifestó como una línea, sino como una red compleja de elementos, relaciones y configuraciones jerárquicas. Finalmente, pudimos observar que tanto el problema de diseño, como las condiciones de su abordaje no han sido previas, sino que se han ido configurando o constituyendo en el avance mismo del proceso proyectual.

\section{Conclusiones}

Con una revisión de los modelos conceptuales y con un relevamiento de las estrategias metodológicas concretas que se desarrollan en las prácticas de los diseñadores, presentamos un modelo preliminar que captura 


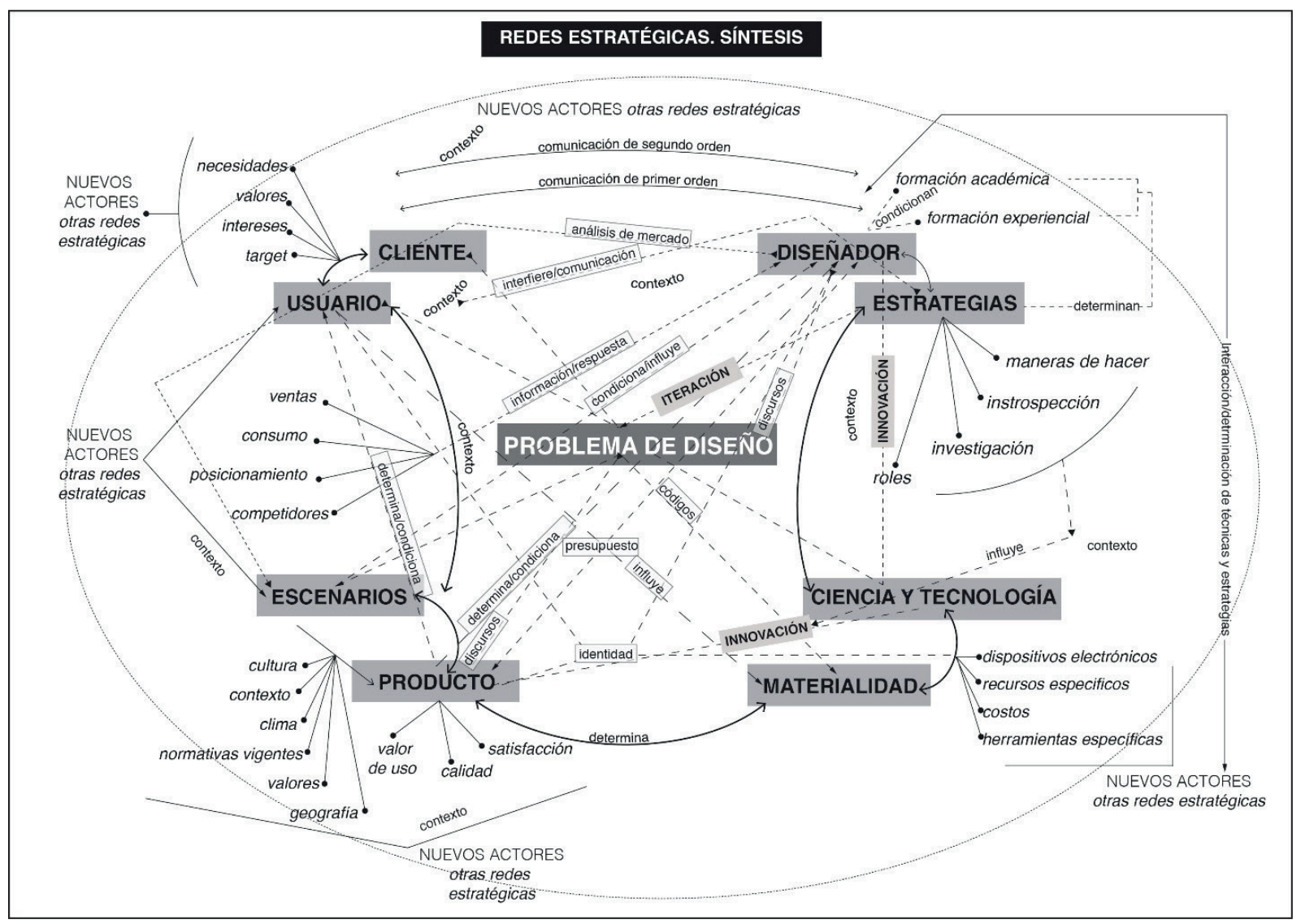

los aspectos principales que conformaron el 'menú' de actores y actantes, configurados como una red estratégica para la toma de decisiones, ver Figura 4.

Además, podemos sintetizar los siguientes resultados:

i) El modelo que se advirtió en el análisis de las prácticas no se relaciona con el 'camino' de fases, etapas o instancias más o menos lineales o secuenciales. Por el contrario, se presentaron múltiples acciones y decisiones consistentes con la configuración de una red compleja de interacciones entre agencias diversas.

ii) Se identificaron aspectos comunes a todos los modelos metodológicos producidos en cada disciplina proyectual, llegando a considerarlas como Invariantes estructurales. Ellas son: problema, usuario, cliente, procesos, condicionantes, demandas, usos, discursos (modelos), contextos. Asociados por ciertas proximidades funcionales, operan como ámbitos o nodos centrales en los procesos y estrategias metodológicas del campo proyectual.

iii) Asimismo, se han identificado procesos comunes a todos los modelos, denominándolos invariantes funcionales. Entre las principales podemos mencionar: entramado, reticulación, conectividad, re-presentación (en red). En términos de Latour y de su ontología del actante-rizoma, estas invariantes funcionales o dinámicas se podrían explicar como la capacidad de agencia o de conectividad que tienen los diferentes aspectos que se consideran en todo proceso de diseño.
Figura 4. Modelo reticular con actores, actantes y discursos de las prácticas de diseño gráfico, de indumentaria y textil. Fuente: Elaboración propia. 
Concretamente sería como pensar en el modo en que "una cosa se vincula con otras cosas y así adquiere su relevancia en el sistema".

iv) Los elementos a los que la red da respuesta se re-constituyen en la misma red. No es un proceso de respuesta a un problema externo ya existente, es un proceso de construcción co-constructiva de problemas, actores, objetos, relaciones, contextos y respuestas. Los elementos de la red se conectan entre sí llevando a configuran nuevas relaciones. Esta conectividad de la red permite (obliga) a que aparezcan elementos de una 'trama oculta' que da sentido al 'objeto complejo' que se va generando.

v) De esta manera el objeto que se diseña en el contexto de una trama metodológica compleja permite o facilita los procesos de innovación, incluso antes de ponerse en uso. Si desde el punto de vista de las lógicas de constitución ya atiende a todos los aspectos y variables del problema, luego le será más fácil evolucionar en la trama praxeológica de la "realidad".

vi) La innovación no es, entonces, un proceso que se dispara con el producto, sino que se juega ya en el mecanismo de constitución. Y su condición de estratégica es una propiedad de su producción en una trama de reticulaciones y conexiones que se producen en la misma red del diseño.

vii) Ante las preguntas: ¿Cómo la red maneja la diversidad y la multiplicidad? ¿es un espacio de posibilidades?. Hemos detectado que la red va construyendo relaciones, interacciones entre sus partes y representaciones de esas interacciones. La inteligencia propia de la red produce una saturación de las relaciones que terminan por estabilizar la red. Ya no se dan tantas posibilidades, sino que se produce una orientación o convergencia en la respuesta. Esta orientación puede adoptar diferentes formatos: Ideas generadoras; pautas, configuraciones, etc.

viii) Las redes son estratégicas porque representan el objeto complejo (múltiple) que abordan. En ese abordaje no siguen un lineamiento arbitrario, sino abierto a los componentes de la red. La misma red tiene un mecanismo que le permite sintetizar lo diverso: a este fenómeno hemos denominado "la inteligencia estratégica de la red". Significa que en algún momento del proceso se saturan sus posibilidades y se orientan hacia una dirección de respuesta coherente.

ix) Hemos detectado que en múltiples situaciones el diseñador hace uso de otras redes no proyectuales que intervienen en el proceso. Por ejemplo, de redes epistémicas, semióticas, psicológicas, etc. Lo que permite pensar en un escenario más complejo de inter-acción entre redes de actores y actantes de múltiples disciplinas.

$x)$ Finalmente, podemos afirmar que las redes que se producen en el diseño generan -además del producto diseñado- una nueva dimensión de conocimientos. Pensamos que esta condición apoya la concepción sostenida por la Teoría del Actor-Red, en la cual "El actor-red no es reductible ni a un simple actor ni a una red. [...] Un actor-red es, simultáneamente, un actor cuya actividad consiste en entrelazar elementos heterogéneos y una red que es capaz de redefinir y transformar aquello de lo que está hecha" (Callon, 1987, p.93). 


\section{Referencias}

Alexander, C. (1964). Notes of the Synthesis of Form. Massachusetts: Harvard University Press.

Archer, L. B., (1984). Systematic method for designers. In Cross, N. (Ed.), Developments in Design Methodology. Chichester UK: Wiley, 57-82.

Archer, B. (1981). A view of the nature of Design Research. In Jackes, R., \& Powell, J. (Eds.), Design: Science: Method. (pp. 30-47). Great Brittain: Westburg House.

Bijker, W., Hugghes, T., \& Pinch, T. (1987). The social construction of technological system. Cambridge, Massachusetts: MIT Press.

Broadbent, G. (1984). Design and Theory Building. En N. Cross, N. (Ed.), Developments in design Methodology. (pp. 277-290). Chichester, UK: Wiley.

Buchanan, R. (1985). Declaration by Design: Rhetoric, Argument, and Demonstration in Design Practice. Design Issues, 2(1), 4-22.

Buchanan, R. (1992). Wicked Problems in Design Thinking. Design Issues, 8(2), 5-21.

Burgos, C. (2010). Dimensiones Epistémicas y Cognitivas en la Enseñanza de las Disciplinas Proyectuales. Arquisur, o(o1), 80-91.

Burgos, C. (2013). La Condición Cognitiva Esencial del Diseño Arquitectónico. Morfología y Dinámica del Proceso de la Acción Proyectual, ADNea, ol(01), 53-62.

Burgos, C., lbarra, A. (2015). Teoría del Diseño: De las lógicas del objeto-problema a las lógicas de los procesos constitutivos. Representaciones, 10(02), 21-45.

Callon, M. (1987). Society in the Making: The Study of Technology as a Tool for Sociological Analysis. In Bijker, W., Hughes, T., \& Pinch, T. (Eds.), The Social Construction of Technological Systems. Cambridge, MA: MIT Press.

Cross, N. (1982). Designerly Ways of Knowing. Design Studies, 2(4), 118-227.

Cross, N. (1984). Developments in Design Methodology. Chichester, UK: John Wiley \& Sons.

Cross, N. (1999). Design Research: A Disciplined Conversation. Design Issues, 15(2), 5-10.

Cross, N. (2007). Designerly Ways of Knowing. Berlin: Birkhäuser Verlag.

Cross, N. (2010). Design Expertise. Design Studies, 31(2), 203-205.

Cross, N. (2011). Design thinking: Understanding how designers think and work. New York: Berg Publishers.

Cross, N. (2008). Engineering Design Methods: Strategies for Product Design (4th ed.). Chichester, UK: Wiley.

Cross, N., Christiaans, H., \& Dorst, K. (1996). Analyzing Design Activity. Chichester, UK: Wiley.

Curry, T. (2014). A theoretical basis for recommending the use of design methodologies as teaching strategies in the design studio. Design Studies, 35(6), 632-646.

Dubberly, H. (2004). How do you Design? A compendium of Models [online]. Disponible en http://www.dubberly.com/ wp-content/uploads/2008/o6/ddo_designprocess.pdf

Farrell, R., \& Hooker, C. (2013). Design, Science and Wicked Problems. Design Studies, 34(6), 681-705.

Frascara, J. (1988). Graphic Design: Fine Art or Social Science?, Design Issues, 5(1), 18-29.

Frascara, J. (2000). Diseño gráfico para la gente: Comunicaciones de masa y cambio social. Buenos Aires: Infinito.

Frascara, J. (2018). Enseñando Diseño: Usuarios, Contextos, Objetivos y Métodos de Investigación. Buenos Aires: Infinito.

Galle, P. (1999). Design as Intentional Action: A Conceptual Analysis. Design Studies, 20(1), 57-81.

Galle, P. (2002). Philosophy of Design: An Editorial Introduction. Design Studies, 23(3), 211-218.

Galle, P., \& Kovacs, L. (1996). Replication protocol analysis: a method for the study of real-world design thinking. Design Studies, 17(2), 181-200.

Gregory, S. A. (1966). The design method. London: Butterworths Press.

Hacking, I. (1996). Representar e intervenir. México: Paidós Ibérica.

Houkes, W. (2006). Knowledge of Artefact Functions. Studies in History and Philosophy of Science (Part A), 37(1), 102-113.

Hutchins, E. (1996). Cognition in the Wild. Cambridge, MA: MIT Press. Jacques, R., \& Powell, J. (1980). Design: Science: Method. Surrey, UK: Westbury House.

Jonas, W. (1993). Design as Problem-Solving? or: Here is the Solution, what was the Problem? Design Studies, 14(2), 157-170.

Jones, J. (1992). Design Methods (2nd ed.). New York: John Wiley \& Sons.

Kimbell, L. (2011). Rethinking design thinking: Part I. Design and Culture, 3(3), 285-306.

Kimbell, L. (2012). Rethinking design thinking: Part II. Design and Culture, 4(2), 129-148.

Kokotovich, V., \& Dorst, K. (2016). The art of 'stepping back': Studying levels of abstraction in a diverse design team. Design Studies, 46, 79-94.

Kroes, P. (2002). Design Methodology and the Nature of Technical Artefacts. Design Studies, 23(3), 287-302.

Latour, B. (1997). On Actor Network Theory: A Few Clarifications. Soziale Welt, 47(4), 369-381.

Lawson, B., \& Dorst, K. (2009). Design Expertise. Oxford Elsevier: Architectural Press.

Margolin, V. (1989). Design Discourse: History, Theory, Criticism. Chicago, IL: University of Chicago Press.

Margolin, V., Morales, B., et al. (2007). Las Rutas del Diseño: Estudios sobre Teoría y Práctica. Buenos Aires: Nobuko. 
Morelli, N. (2007). Social Innovation and New Industrial Contexts: Can Designers Industrialize Socially Responsible Solutions? Design Issues, 23(4), 3-21.

Morin, E., et al. (2003). Educar en la era planetaria. Barcelona: Gedisa.

Munari, B., \& Rodríguez, C. (1989). ¿Cómo nacen los objetos? Madrid: Gustavo Gili.

Munari, B. (2006). Design e comunicação visual: contribuição para uma metodologia didática. São Paulo: Martins Fontes.

Newman, M. (2003). The Structure and Function of Complex Networks. Siam Review, 45(2), 167-256.

Pierri, P. (2017). Decentralising Design. Raising the Question of Agency in Emerging Design Practice. The Design Journal, 20 (sup1), 951-969.

Roth, S. (1999). The State of Design Research. Design Issues, 15(2), 18-26.

Schön, D., \& Wiggins, G. (1992). Kinds of Seeing and their Functions in Designing. Design Studies, 13(2), 135-156.

Simon, H. (1988). The Science of Design: Creating the Artificial. Design Issues, 4(1-2), 67-82.

Simon, H. (1996). The Sciences of the Artificial (3rd ed.). Massachusetts: MIT Press,

Visser, W. (2006). The Cognitive Artifacts of Designing. Mahwah, NJ: Lawrence Erlbaum Associates Publishers.

Visser, W. (2009). Design: One, but in Different Forms. Design Studies, 30(3), 187223. 